\title{
THE ROLES OF SLIP GEOMETRY AND HARDENING BEHAVIOR IN INTERGRANULAR TOUGHNESS
}

DOE/ER/451 $98-8$

\section{Progress Report} \\ C. Laird/J. Bassani \\ for the Period January 15, 1992 through January 15, 1993 \\ University of Pennsylvania \\ Dept. of Materials Science \& Engineering \\ Dept. of Mechanical Engineering \\ Philadelphia, PA 19104
}

DE93 005647

September 1992

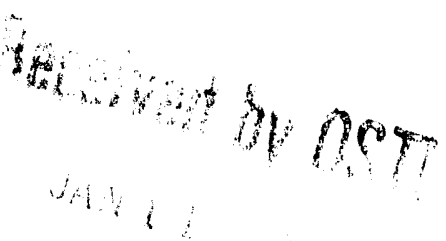

Prepared for

\section{THE UNITED STATES DEPARTMENT OF ENERGY AGREEMENT NO: DE-FG02-85ER-45188}

DISCLAIMER

\begin{abstract}
This report was prepared as an account of work sponsored by an agency of the United States Government. Neither the United States Government nor any agency thereof, nor any of their cmployees, makes any warranty, express or implied, or assumes any legal liability or responsibility for the accuracy, completeness, or usefulness of any information, apparatus, product, or process disclosed, or represents that its use would not infringe privately owned rights. Reference herein to any specific commercial product, process, or service by trade name, trademark, manufacturer, or otherwise does not necessarily constitute or imply its endorsement, recommendation, or favoring by the United States Government or any agency thereof. The views and opinions of authors expressed herein do not necessarily state or reflect those of the United States Government or any agency thereof.
\end{abstract}




\section{EXPERIMENTAL STUDIES}

\section{Cyclic Response of Polycrystalline Copper}

During 1992 w completed our project on polycrystalline cyclic plasticity inherited from last year and the student involved proceeded successfully to his $\mathrm{PhD}$ degree. We have unravelled the roles of a wide variety of factors which influence the polycrystalline cyclic stress-strain curve of fcc metal, namely: loading mode, ramp pretreatment, deformation history, grain size, and texture, and we can now explain the structure sensitivity of the cyclic stress strain curve. This work has culminated in a series of seven papers (see \#'s 4-10 in the list), in which the collaboration of A.D. Rollett of Los Alamos National Lab with respect to texture questions has been invaluable.

The role of boundaries, per se, has not yet been elucidated, but theoretical developments from our bicrystal project have been extremely useful in explaining several aspects of polycrystalline cyclic plasticity. For example, consider the role of twins, which generally favor the formation of persistent slip bands (PSB's) adjacent to them. Figure 1 shows the Schmid faciors of all (111) type crystal systems for a crystal with a [123] axis, i.e., a single slip orientation (side 1), adjacent to its twin (side 2). The coarsely-shaded bars indicate the Schmid factors for the two crystals treated in isolation from each other. The finer-shaded bars show the Schmid factors as influenced by the interactive effect of the adjacent crystal, treated on the basis of elastic behavior. Thus the [123] crystal is changed to double slip behavior and its twin is changed from a double slip to single slip orientation. Since multi-slip conditions promote the transition of loop patches to PSB"s, we have been able to explain those intertwin situations which give rise to preferred PSB formation. Fig. 2 shows how for a [111] oriented crystal, using the same codes as in Fig.1, the Schmid factors can be increased by the presence of the twin and the multiplicity of slip altered. Nevertheless, textures containing grains oriented for multiple slip do show 
more pronounced hardening. On this basis, we have shown that the "anomalous" increased cyclic hardening levels of large-grained copper is not actually a grain size effect, but a [111][100] texture effect associated with the annealing procedures needed to form large grains. 'ive have more recently supported our interpretation by forming large grain structures with $[111] /[100]$ textures, and then producing specimens with different textures by cutting them trom the stock at various angles to the rolling/texture direction. The stress levels shown by the cyclic stress-strain curves of such specimens are appropriately reduced by the extent that the "new" textures provide opportunity for single slip. These results are now being prepared for publication.

\section{Bicrystal Experiments and Boundary Theory}

The development work initiated last year in selecting and preparing bicrystals has proceeded extremely well in spite of the difficulties associated with bicrystals and we have begun the prosed experimentation on how near boundary deformation affects boundary fracture mechanisms. As a demonstration of success in technique, Figure 3(a) shows the corresponding stress-strain curves of a single crystal oriented for single slip and of a bicrystal with a transverse boundary having one crystal similarly oriented; Fig. 3(b) shows a bicrystal which has been pulled to fracture, and the excellent integrity of the bicrystal boundary seen just to the right of center. Necking and ductile rupture of one crystal has occurred off-field-of view to the right and the boundary has survived intact.

An important aspect chosen for study is the effect of incompatibility of deformation on the cracking behavior at a boundary. In that regard, a model has been developed to estimate the elastic stresses that arise at the boundary of a bicrystal (the same model was used for the twin boundary problem described above) because of incompatibility in elastic deformation. This model has been based on the 
elasticity solutions developed by the ech workers Gemperlova et al., and Bassani and $\mathrm{Qu}$, for the piecewise homogeneous stresses at the boundary of two generally anisotropic solids, where a homogeneous stress field is assumed to exist close to the boundary and far away from the edges of the specimen. Under the appropriate boundary conditions, this solution is exact for infinite media and it may apply in a Saint Venant's sense when the bicrystal is large enough. For the geometry of the specimen selected for our studies, an edge effect must be significant because of the square cross section and limited size. Nevertheless, this elastic solution has been used to predict slip behavior, with good agreement with experiment, for bicrystal boundaries fairly similar to those chosen by use (Paidar et al., Acta Met., 34 (1986) 2277 and Mat. Sci. Eng., A137 (1991) 69). The first result obtained here by using this model is that the elastic deformation in tension of copper bicrystals with symmetrical tilt boundaries about the [110] axis is compatible, i.e., the compatibility conditions are all satisfied.

In view of this result it was decided to use an asymmetrical tilt boundary about the [110] axis in order to produce in compatibility stresses. The model to compute the stresses was then modified to calculate the incompatibility stresses as a function of the misorientation angle of the crystals with respect to their original crystallographic axes, according to the geometry shown in Fig. 4.

The results of the calculations are shown (in part) in Figures 5 and 6 . In the graphs shown, the angles $\mathbf{a}_{1}$ and $\mathbf{a}_{2}$ are expressed in degrees and they represent twice the misorientation angle. Three dimensional plots are shown in Fig. 6, two dimensional plots in Fig. 5.

These plots demonstrate that the maximum incompatibility stresses occur for orientations close to $180^{\circ}$. Unfortunately, these represent multiple slip orientations of the crystals, i.e., the corners of the stereographic triangle, for which the slip is already multiple, and which is undesirable in an experiment where the aim is to 
induce multiple slip in unpredicted conditions. Therefore, further misorientations were explored. In this exploration, one of the crystals was maintained with fixed orientation equal to that of the $\Sigma 9$ boundary selected by Rice et al., and selected by us also because it offered interesting possibilities for deformation/fracture interaction. Then the stresses were recalculated as the orientation of the other crystal was made to vary. The maximum incompatibility stresses were found to occur for $\alpha=180^{\circ}$, as expected, but to avoid crystal orientations in which the slip would be multiple, but where the incompatibility would still be significant, a misorientation angle for the second crystal was chosen as $141^{\circ}$.

Calculations such as these have been extremely useful for refining our bicrystal experiments, and providing a base for interpretation of the deformation and fracture behavior. The results of the calculations have now been confirmed and elaborated by finite element technique. For example, the effect of the finite size of the specimen has now been explored. Fig. 7 shows the extent to which the stresses are localized at the boundary as a function of the length/width ratio of the gauge length (see Fig. 4 for a description of the coordinate system).

\section{Interfacial Discontinuities}

This analytical study is concerned with the relationship between the stress and strain fields on one side of an interface with those on the other. We have extended some ideas of Hill's for isotropic bimaterials with perfect bonding to anisotropic bimaterials with imperfect bonding whicit is introduced to model distinct interfacial properties. The main idea is rooted in partitioning second-order and fourth-order tensors into interior (e.g., non-traction) and exterior (e.g., traction) parts. For example, if the interface normal is in the $x_{3}$ direction, the the interior part of the displacements and stresses are, respectively 


$$
\begin{aligned}
\mathbf{u}_{1} & =\left(u_{1}, u_{2}, 0\right), \\
\boldsymbol{\sigma}_{1} & =\left[\begin{array}{ccc}
\sigma_{11} & \sigma_{12} & 0 \\
\sigma_{12} & \sigma_{22} & 0 \\
0 & 0 & 0
\end{array}\right]
\end{aligned}
$$

while the corresponding exterior parts are

$$
\begin{aligned}
\mathbf{u}_{\mathrm{E}} & =\left(0,0, u_{3}\right), \\
\boldsymbol{\sigma}_{\mathrm{E}} & =\left[\begin{array}{ccc}
0 & 0 & \boldsymbol{\sigma}_{13} \\
0 & 0 & \boldsymbol{\sigma}_{23} \\
\sigma_{31} & \sigma_{32} & \boldsymbol{\sigma}_{33}
\end{array}\right]
\end{aligned}
$$

For an arbitrary orientation of the interface one can write

$$
\begin{aligned}
& \sigma_{1}=T: \sigma=\sigma: T, \\
& \sigma_{E}=N: \sigma=\sigma: N
\end{aligned}
$$

where in terms of the unit normal $\mathbf{n}$ to the interface

$$
N_{i j k l}=\delta_{i k} n_{j} n_{1}+\delta_{j l} n_{i} n_{k}-n_{i} n_{j} n_{k} n_{l}
$$

and

$$
T_{i j k l}=I_{i j k l}-N_{i j k l}
$$

with

$$
I_{i j k l}=\frac{1}{2}\left(\delta_{i k} \delta_{j l}+\delta_{i l} \delta_{j k}\right)
$$

In general, the exterior part of the stress must be continuous and, for perfect bonding, the interior part of the strain must be continuous.

The important new result is derived from also partitioning the fourth-order tensor of elastic compliances where $\varepsilon=\mathbf{M}$ : $\sigma$ as follows

$$
\begin{aligned}
& \mathbf{M}_{\mathrm{I}}=\mathbf{T}: \mathbf{M}: \mathbf{T}^{\mathrm{T}} \\
& \mathbf{M}_{\mathrm{E}}=\mathbf{N}: \mathbf{M}: \mathbf{N}^{\mathrm{T}} \\
& \mathbf{M}_{\mathrm{IE}}=\mathbf{T}: \mathbf{M}: \mathbf{N}+\mathbf{N}^{\mathrm{T}}: \mathbf{M}: \mathbf{T}
\end{aligned}
$$


From these three partitions, the jump in the interior part of the stress can be related to the jump in the interior part of the strain according to

$$
\left(M_{\mathrm{I}}^{(2)}+\mathbf{M}_{\mathrm{E}}^{(2)}\right): \sigma_{\mathrm{I}}^{(2)}-\left(\mathbf{M}_{\mathrm{I}}^{(1)}+\mathbf{M}_{\mathrm{E}}^{(1)}\right): \sigma_{\mathrm{I}}^{(2)}+\left(\mathbf{M}_{\mathrm{lE}}^{(2)}+\mathbf{M}_{\mathrm{lE}}^{(1)}\right): \sigma_{\mathrm{E}}=\varepsilon_{1}^{(1)}-\varepsilon_{\mathrm{I}}^{(2)}
$$

where superscripts 1 and 2 denote the regions on either side of the interface.

The significance of this result for the proposed research is that we can now explore strain localization in the neighborhood of interfaces, e.g., grain boundaries. It turns out that given continuity of the exterior stress and either continuity of the interior strain or some interface traction/displacement-jump relation, the additional equilibrium assumption of $\dot{\sigma}^{(2)}=-\sigma^{(1)}$, which agrees well with finite element solutions for the fields away from free edges. The analysis will be as follows. Given an exterior loading on a bicrystal and the current state of multiple slips in each crystal, the response to a continued increment in loading can be calculated using the above relations where stresses, strains and compliances are interpreted in the incremental sense. Then, at each stage in the loading, we will explore strain localizatior following the development of Mohan and Bassani (1992) and Bassani (1992), as well as studying interface localizations following Needleman and Ortiz and others.

\section{Shear Localization in Single Crystals}

The work detailed in this section as well as the earlier hardening studies supported by this grant have been summarized in a recent invited paper in the Advances in Applied Mechanics (Bassani, 1992).

In this study a three-dimensional FCC crystal is loaded in uniaxial tension from an unstressed state. The tensile axis is assumed to remain aligned with a given material fiber so that the lattice rotates with respect to that axis during 
loading. The complete evolution of slip activity, including secondary slips, of hardening according to the theory developed in Bassani and Wu (1991), and of the instantaneous moduli entering the bifurcation condition (see, Mohan and Bassani, 1991; Bassani, 1992), are determined throughout the homogeneous part of deformation. Both coarse slip band formation at the transition from stage I to stage II for "single-slip" orientations and macroscopic shear band formation in stage III for "double-slip" orientations are predicted.

The parameters in the hardening law are taken to describe copper with $q=0$ (i.e., no latent hardening); most have been given in Bassani and Wu (1991), while additional parameters that distinguish the saturation hardening-rate in stages I and III are $h_{a}^{I}=15 \tau_{0}$ and $h_{d}^{I I I}=0.075 \tau_{0}$. For these calculations, an efficient numerical scheme has been utilized to integrate the constitutive equations under mixed boundary conditions and to determine the critical state, i.e. the first bifurcation from (see Ortiz et al. 1987). Since the complete stress-strain behavior up to bifurcation is calculated, at that instant not only are $(h / \sigma)_{c r}$ and the shear plane orientation determined, so are the overall stress and strain as well as the evolution of hardening on each slip system. Details of this calculation will appear in Mohan and Bassani (1992).

To set the stage for these three-dimensional bifurcation results, we first recall the macroscopic shear band analysis of Peirce (1983) which accounted for the actual 3-D geometry, with symmetric slip restricted to the primary and conjugate systems. In fact, this is the geometry from which Asaro's approximate 2-D double-slip model is derived. An essentially identical bifurcation analysis for the 3-D configuration predicted the formation of a (macroscopic) shear band at very small values of $\left(H / \sigma_{22}\right)_{c r}$ much smaller than in the 2-D model, and there was little or no misorientation between the primary slip system and the shear band. Based upon the calculations presented below, we conclude that small secondary slips also are 
very important in the 3-D symmetric slip case. Why they apparently are not as important in the 2-D model is not clear, but it does raise questions about the validity of this model in the case of symmetric double slip.

\section{Coarse Slip Bands}

Coarse slip bands are observed early in uniaxial deformations under "singleslip" orientations. Three results for two orientations are plotted in Fig. 8, where the terminus of each curve corresponds to the instant of bifurcation. The curve for orientation 1 assumes elastic isotropy $\left(E=10^{4} \tau_{0}\right.$ and $v=0.3$ ), while for orientation 2 , results for both elastic isotropy and anisotropy are given. First, we note that the localization condition for these single-slip orientations is satisfied at small overall logarithmic strains on the order of $1 \%$. Furthermore, the critical plane orientation deviates very slightly, on the order of $0.001^{\circ}$ as Chang and Asaro (1981) and others have observed. These results are summarized in Table 1, which also includes the ratio of the instantaneous hardening on the primary system (B4) to the overall applied stress. Since the strains are small, the term $h_{a}^{I}$ dominates.

For each of the three cases in Fig. 8 and Table 1, secondary slips initiate before bifurcation. Recall that in pure single slip, for Schmid-type behavior the slip system hardening must go to zero for the first bifurcation. The variation of the hardening moduli on the primary as well as the secondary systems are plotted in Figs. 9a-c; the arrows indicate when the secondary system(s) is activated. For case 1, one secondary system $(\mathrm{C} 1)$ is activated when the overall strain reaches $60 \%$ of the bifurcation strain. For cases $2 \mathrm{i}$ and 2a, two secondary slip systems (A3) and (B5) are activated, and just after that the localization criterion is met. Note that $h_{\mathrm{B} 4}$ decreases $: 0$ a level that is three orders of magnitude less than the hardening rates on the secondary systems. 
The important role of (small) secondary slips is now clearly demonstrated for the formation of coarse slip bands. In the examples given, these form during the beginning of the transition from stage I to stage II hardening when slip interactions are complex. Their role has been suggested from experimental observations as well (see, for example, Basinski and Basinski 1979; Kulhmann-Wilsdorf and Laird 1980; and Basinski and Basinski 1992), but further investigation is needed. This small slips and high hardening rates are difficult to detect, and the latter can be mistaken for elastic as Kulhmann-Wilsdorf (1989) has suggested.

\section{Macroscopic Shear Bands}

Macroscopic shear bands are observed at relatively high levels of overall strain. Four results for a symmetric "double slip" orientation are plotted in Fig. 10, where the terminus of each curve corresponds to the instant of bifurcation. Each curve corresponds to the sime initial orientation [112] with different elastic assumptions and values of the saturation slip parameter $\gamma_{0}^{\prime \prime I}$. Basically, with a given value of $h_{d}^{\prime \prime \prime}$ the larger the value of $\gamma_{0}^{I I I}$, the larger the critical strains which, for the material parameters chosen, are on the order of 0.1 , i.e. an order of magnitude larger than that for the coarse slip bands. Furthermore, the critical plane orientation deviates by $2^{\circ}$ which is similar to the surface observations Chang and Asaro (1981). The results are summarized in Table 2, which includes the ratio of the instantaneous hardening on the primary systems (B4) and (C1) to the overall applied stress.

For each of the four cases in Fig. 10 and Table 2, secondary slips initiate before bifurcation. Recall that in Peirce's (1983) 3-D double slip calculation for Schmid-type behavior, $\left(h / \sigma_{c r}\right)$ is predicted to be very small, much lower than the value of 0.65 predicted in the double slip model; moreover, the shear band is essentially oriented with the primary slip systems in disagreement with Chang and Asaro's (1981) 
observations and the results summarized in Table 2. The variation of the hardening moduli on the primary as well as the most-highly strained secondary systems (A3) and (B5) are plotted in Figs. 11a-d. Four other secondary systems - (A2), (C5), (B2) and (C4) - are activated before bifurcation, but their slips are one to two orders of magnitude smaller than those other two secondary systems - (A3) and (B5). As in the case of coarse slip bands, since the secondary slips are very small, the hardening rates on the secondary systems are three orders of magnitude greater than those on the primary systems.

Once again, the important role of (small) secondary slips is demonstrated, this time for the formation of macroscopic slip bands. In the examples given, these form during stagie III hardening. The only apparent difference between the calculation presented here and that of Peirce (1983) is the role of secondary slips. 


\section{EDUCATION}

During the course of this year's research, the following students have been supported:

L. Llanes, P. Peralta (Materials Science and Engineering)

Llanes has obtained the degree of $\mathrm{PhD}$ in Materials Science during the year and proceeded to a post-doctoral position at the University of Barcelona for one year. Peralta passed his PhD qualifying exams at the top of his class.

Chichung Wei (Mechanical Engineering and Applied Mechanics)

Wei has passed his PhD qualifying exan:s and is now studying interfacial discontinuites and strain localization :

Dr. R. Mohan (Mechanical Engineering and Applied Mechanics)

Dr. Mohan completed a year's post-doctorate study supported by this grant and is now a employed full-time at Battelle. 


\section{PUBLICATIONS RESULTING FROM THE SPONSORED RESEARCH}

1,2. T. Wu, J.L. Bassani and C. Laird, "Latent Hardening of Single Crystals - Part I. Theory and Experiments. Part II - Constitutive Equations", Proc. Roy. Soc. of London, A435, 1-19 and 21-41 (1991).

3. T-Y. Wu, J.L. Bassani and C. Laird, "Hardening of Single Crystals under Cyclic Deformation", submitted to Int. J. of Plasticity.

4. L. Llanes and C. Laird, "The Role of Annealing Twin Boundaries in the Cyclic Deformation of FCC Materials", Mat. Sci. Eng., A155 (1992).

5. L. Llanes and C. Laird, "Effect of Loading Mode on the Cyclic Response and the Associated Substructure of Polycrystalline Copper in the High Cycle Regime", Fat. Fracture Eng. Materials \& Structures, in press (1992).

6. L. Llanes and C. Laird, "Substructure Evolution of Copper Polycrystals under Different Testing Conditions: Conventional Strain Control and Ramp Loading", Mat. Sci. Eng., in press (1992).

7. L. Llanes, A.D. Rollett and C. Laird, "Effect of Ramp-Treatment on the Cyclic Stress Strain Curve of Small Grained Copper", submitted to Mat. Sci. Eng. , 1992.

8. L. Llanes, A.D. Rollett, J.L. Bassani an ' C. Laird, "Effect of Grain Size and Annealing Texture on the Cyclic Response and the Substructure Evolution of Polycrystalline Copper", in preparation.

9. L. Llanes, J.L. Bassani and C. Laird, "Description of the Cyclic Response of Polycrystalline Copper in Terms of a Composite-Grain Model and the Cyclic Behavior of Copper Single Crystals', in preparation.

10. L. Llanes and C. Laird, "Effect of Ramp Treatment on the Cyclic Response of Coarse-Grained Copper", in preparation.

11. J. L. Bassani, "Plastic Flow in Crystals," Advances in Applied Mechanics, vol. 30, in press, 1993.

12. J. L. Bassani and J. Qu, "Interfacial Discontinuities and Piecewise Uniform Fields in Anisotropic Bimaterials," in prepartation.

13. R. Mohan and J. L. Bassani, "The Influence of Hardening on Strain Localization in Single Crystals," in preparation. 
Table 1. Three-dimensional coarse slip band calculation.

\begin{tabular}{|c|c|c|c|c|}
\hline & Elastic & Misori. & $\left(h_{\mathrm{B} 4} / \sigma\right)_{\mathrm{cr}}$ & $\varepsilon_{\mathrm{cr}}$ \\
\hline 1 & Isotropy & $0.001^{\circ}$ & 0.95 & $0.7 \%$ \\
$2 \mathrm{i}$ & Isotropy & $0.001^{\circ}$ & 0.47 & $1.1 \%$ \\
$2 \mathrm{a}$ & Anisotropy & $0.002^{\circ}$ & 0.28 & $1.3 \%$ \\
\hline
\end{tabular}

Table 2. Three-dimensional macroscopic shear band calculation.

\begin{tabular}{|c|c|c|c|c|c|}
\hline & $\gamma_{0}^{\text {II }}$ & Elastic & Misori. & $\left(h_{\mathrm{B} 4} / \sigma\right)_{\mathrm{cr}}$ & $\varepsilon_{\mathrm{cr}}$ \\
\hline 1 & 0.1 & Isotropy & $2.05^{\circ}$ & 0.034 & $12.1 \%$ \\
2 & 0.075 & Isotropy & $2.03^{\circ}$ & 0.035 & $9.3 \%$ \\
3 &. .075 & Anisotropy & $19^{\circ}$ & 0.032 & $9.7 \%$ \\
4 & 0.15 & Isotropy & $19^{\circ}$ & 0.031 & $18 \%$ \\
\hline
\end{tabular}




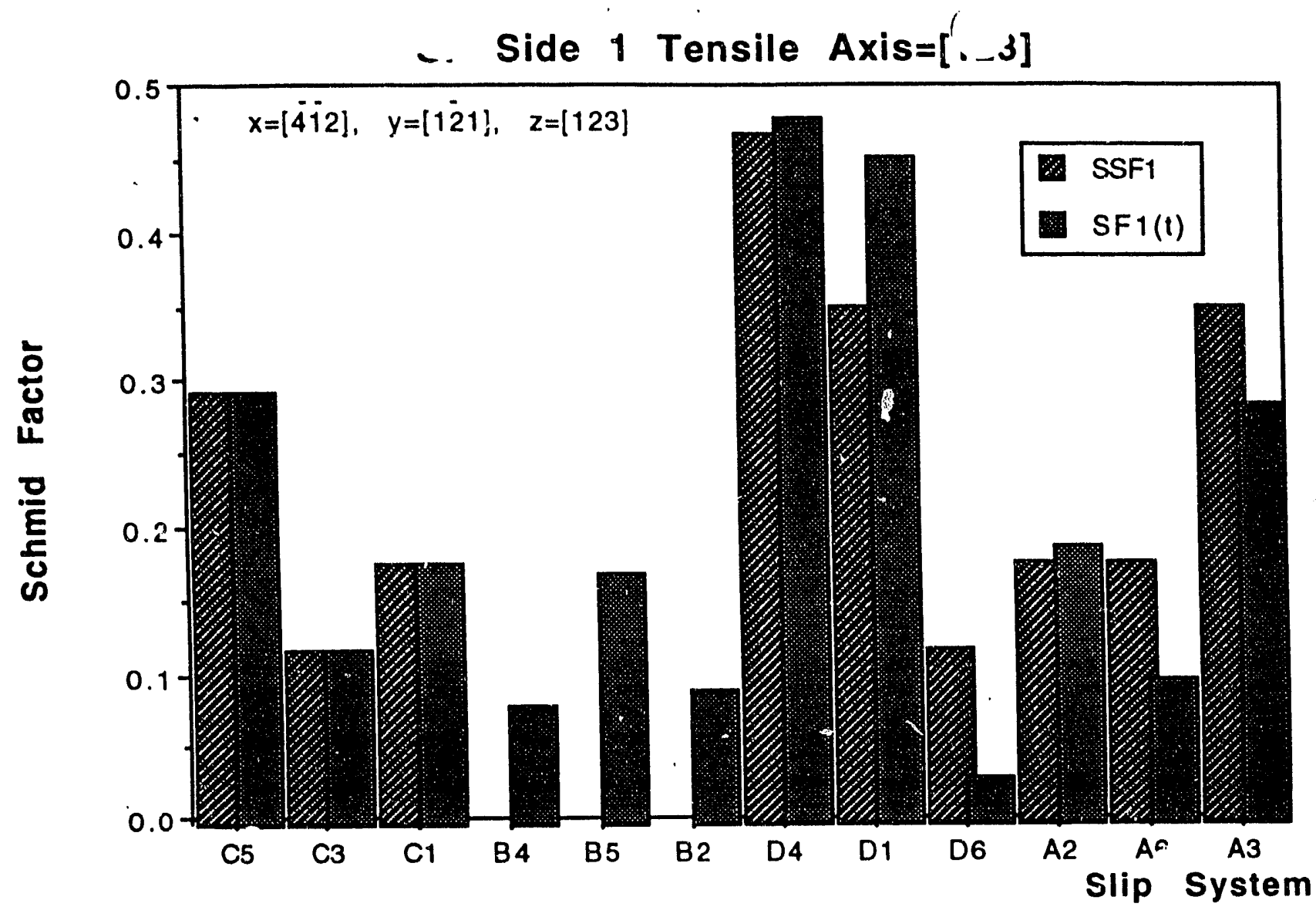

SF Side 2 Tensile Axis $=\left[\begin{array}{lll}-1 & 10 & \overline{5}\end{array}\right]$

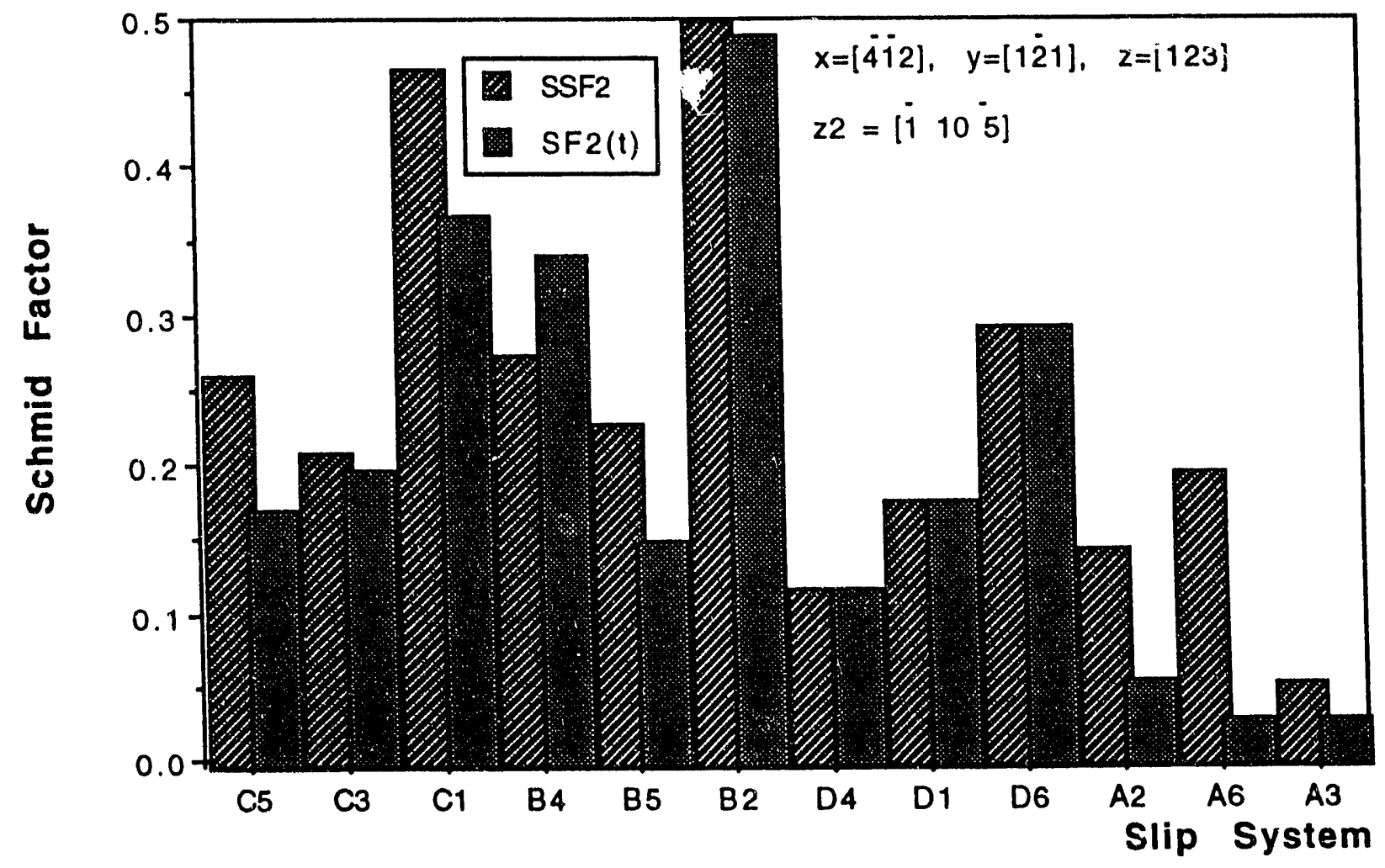

$F \in G .1$ 


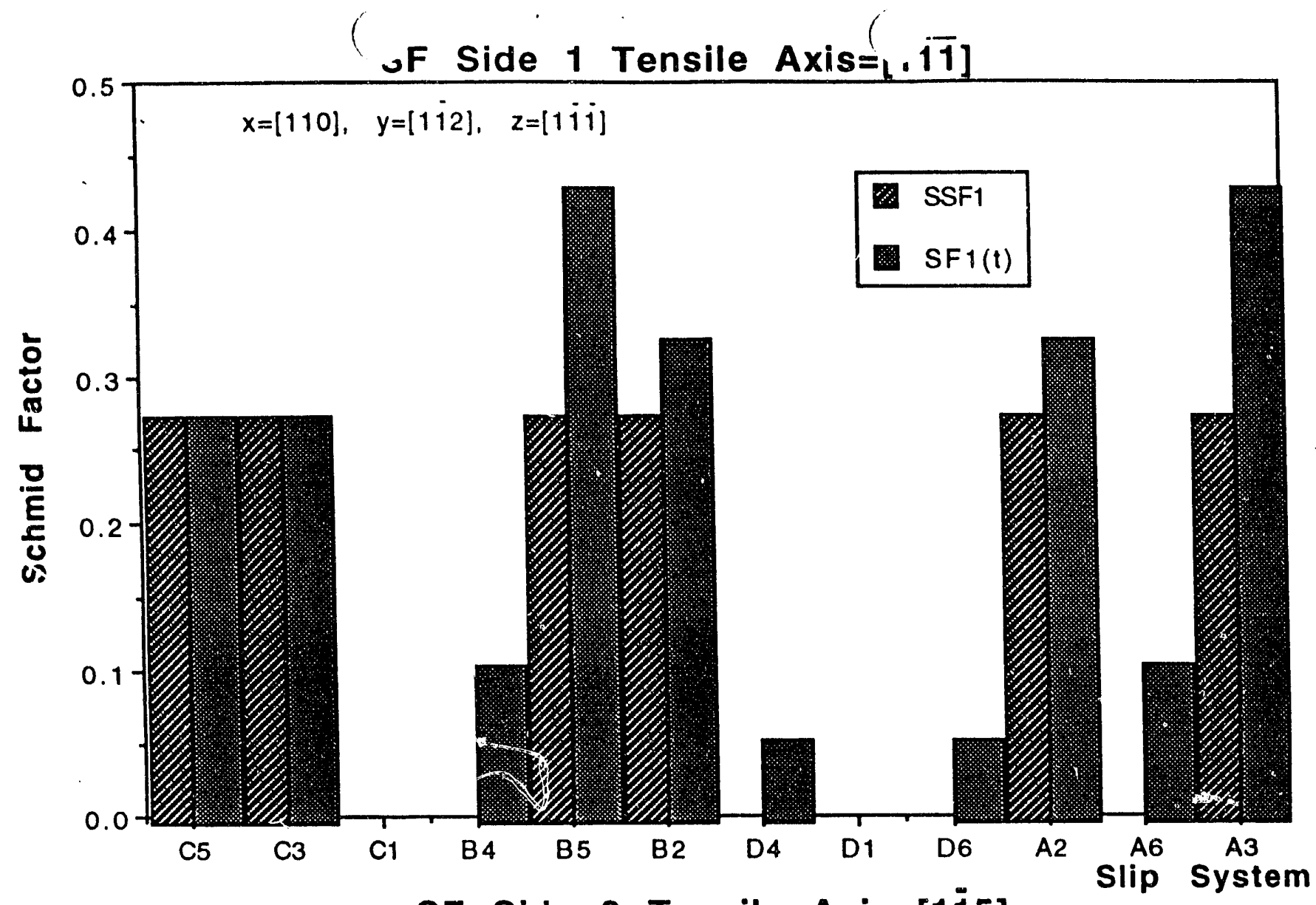

SF Side 2 Tensile Axis $=[115]$

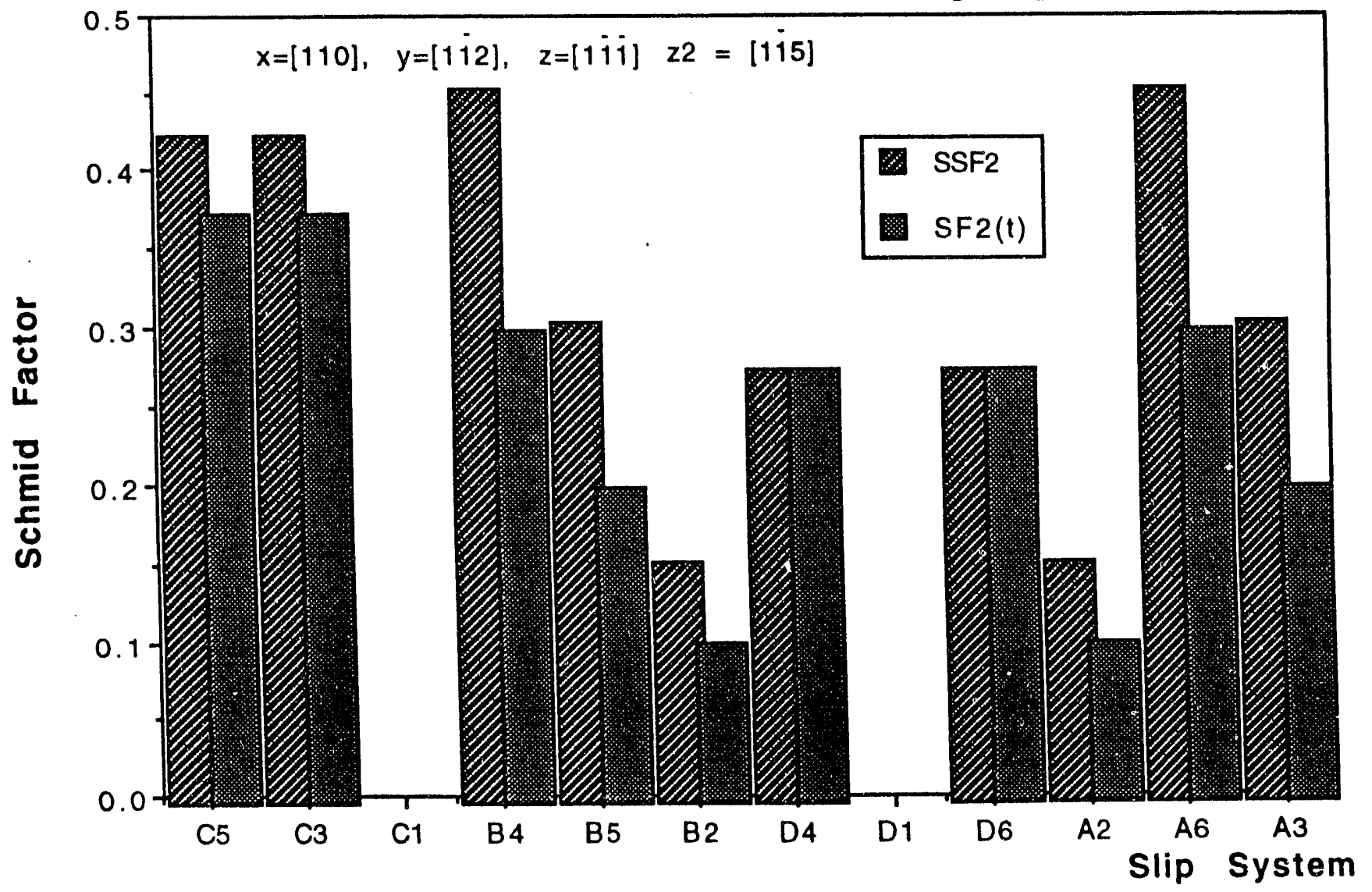

$F \in \cdot 2$. 
Approx. Strength-Strain Curve (<149> Bi-xtal) \& Stress-Strain curve (<149> Single $x$ tal)
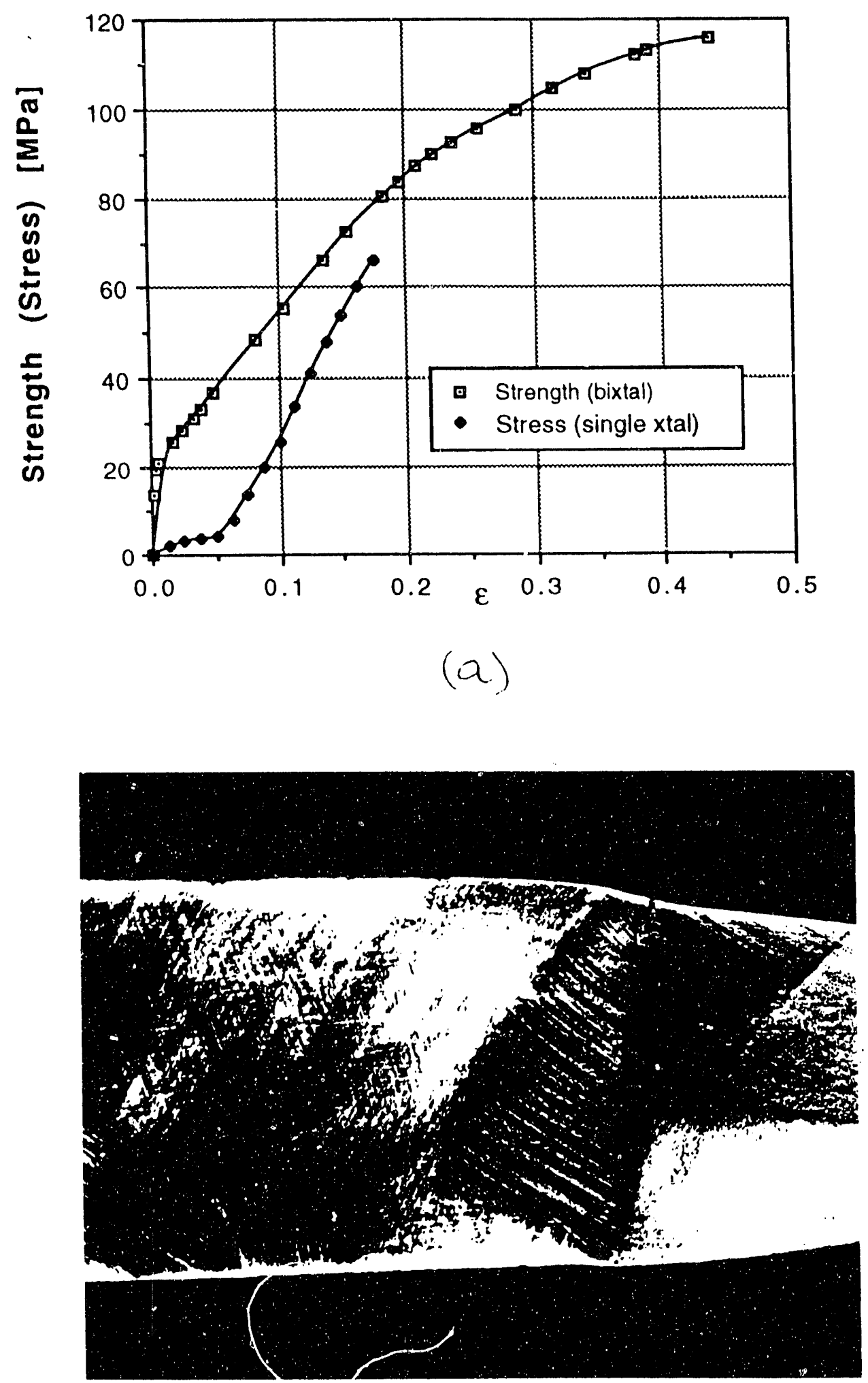

$(\theta)$

$F G . ?$ 


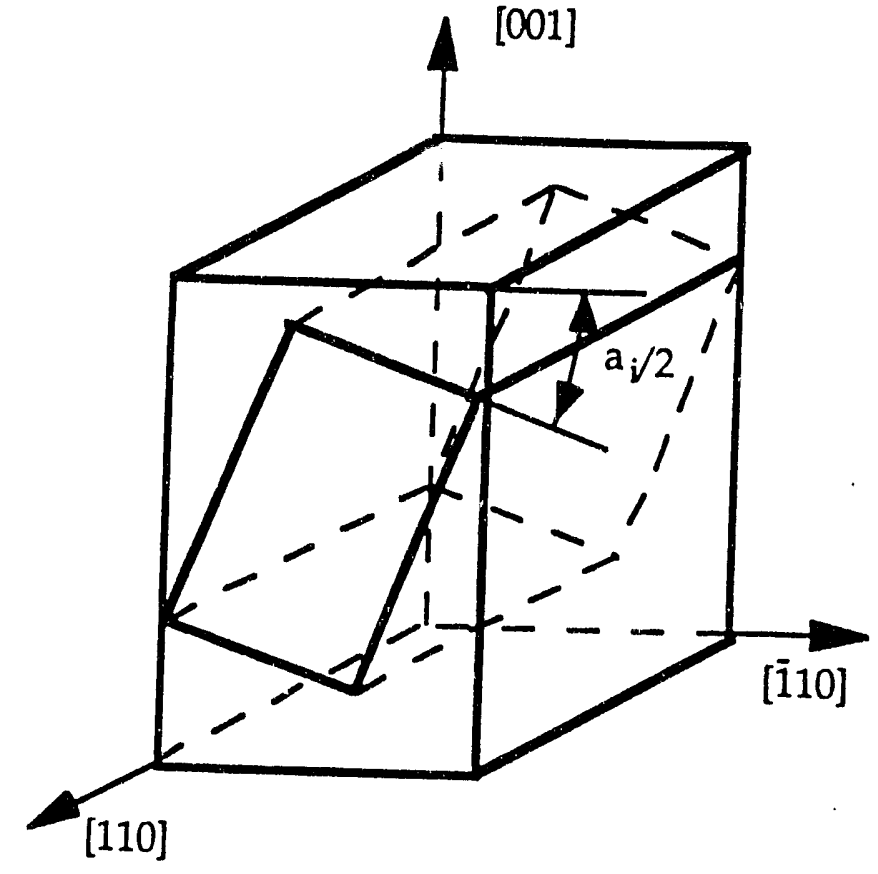

(a)

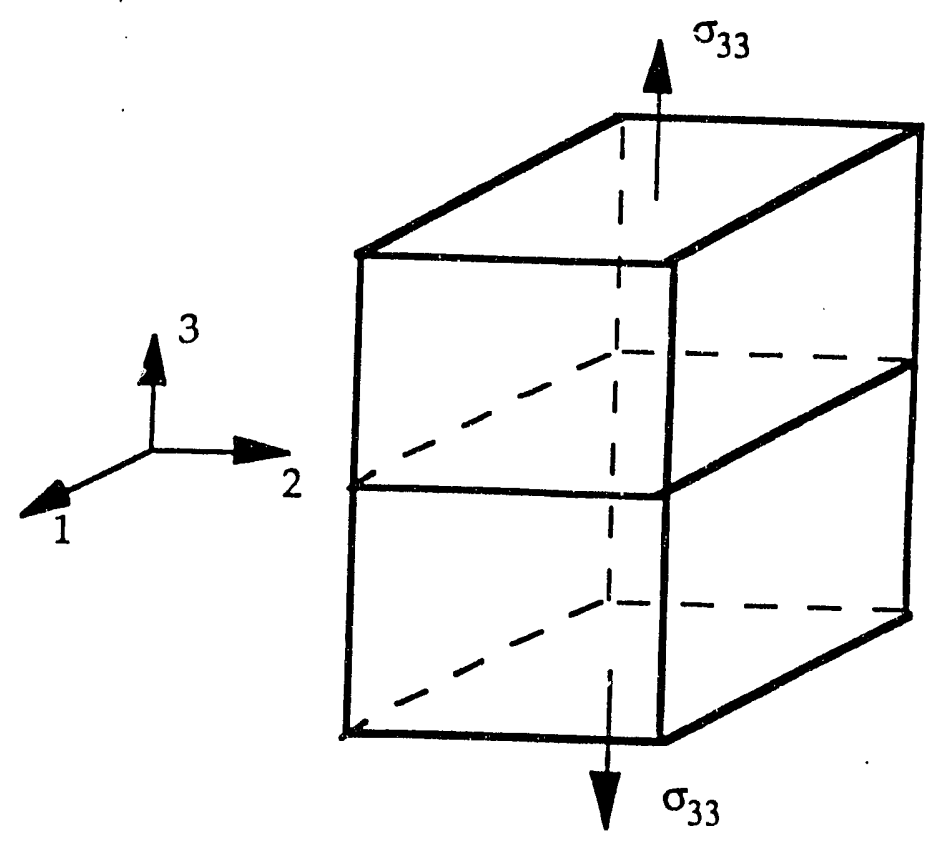

(b)

Fig. 4. Geometry used to calculate the incompatibility stresses. (a) Definition of the misorientation angle $a_{i}$. (b) Coordinate system for the stresses in the bicrystal. 
Sigma 1 vs Angle

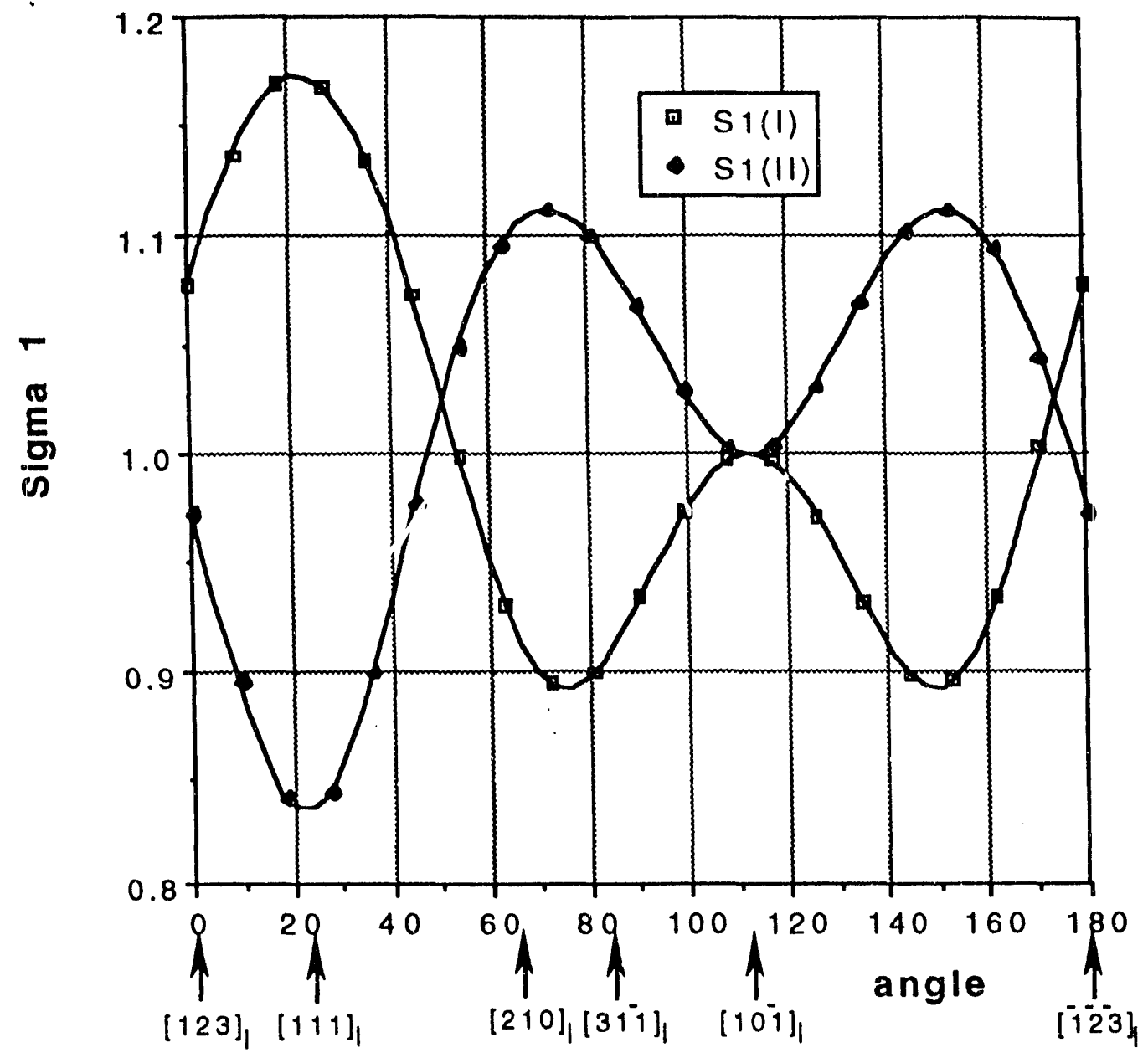

Sigma 3 vs Angle

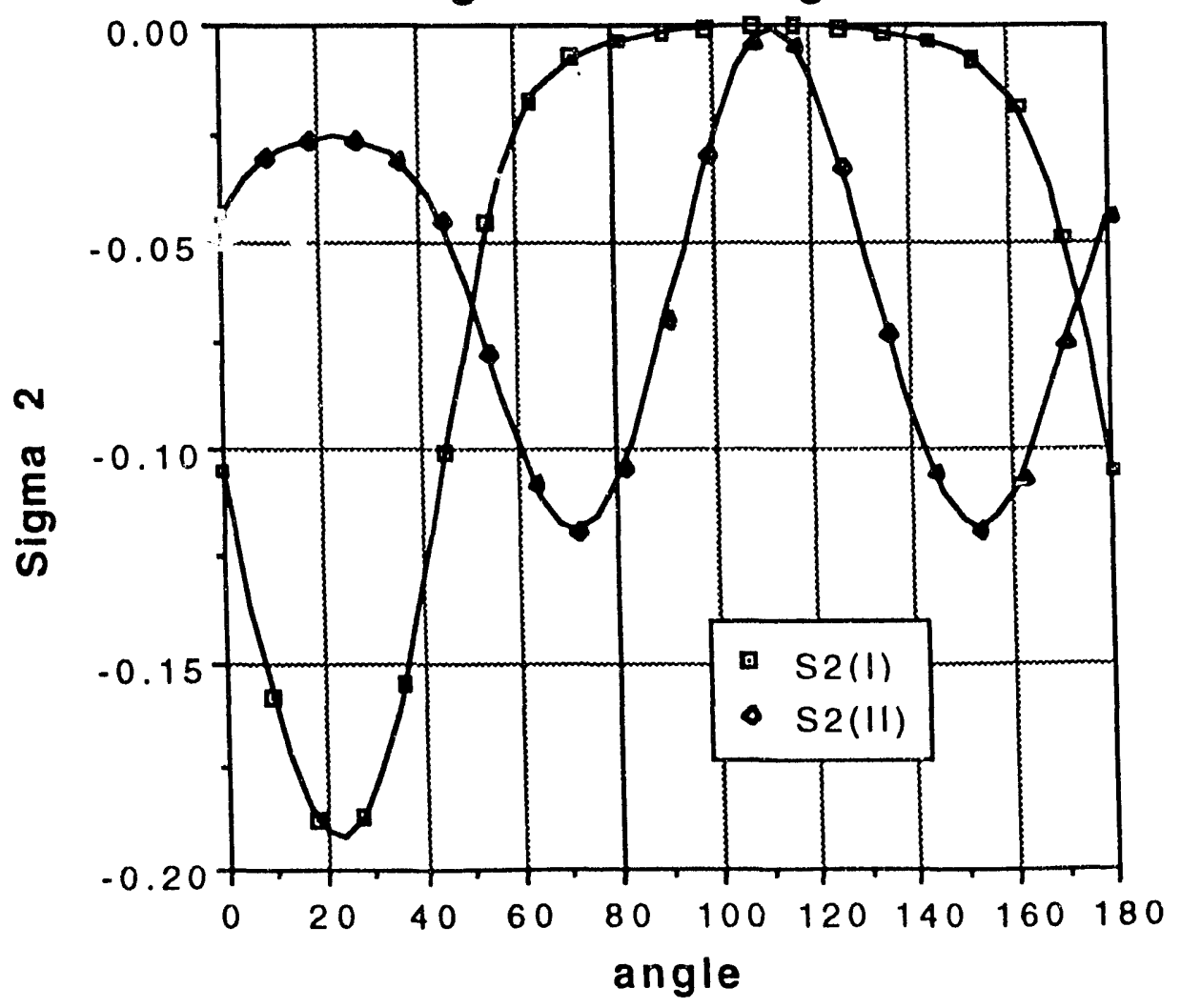

Fic. s 


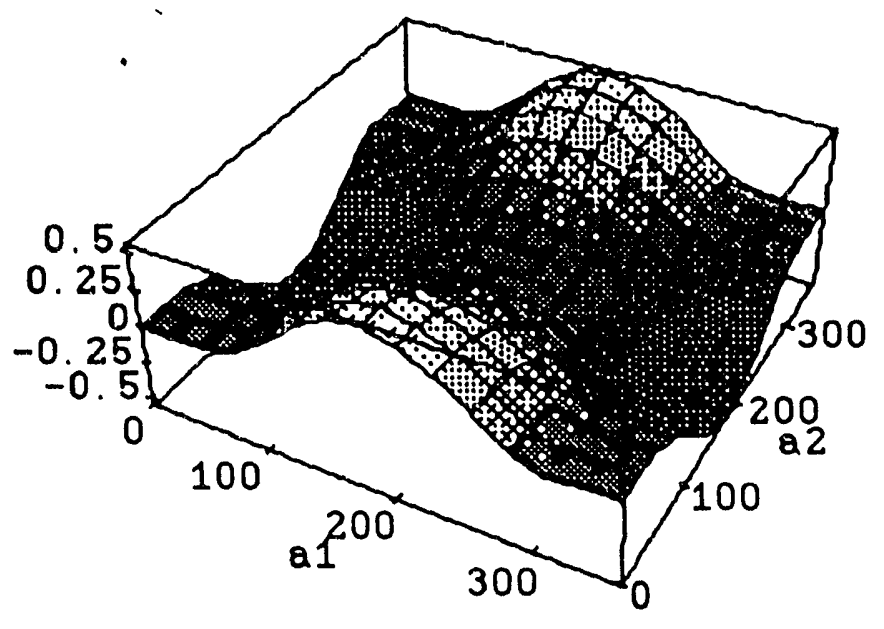

(a)

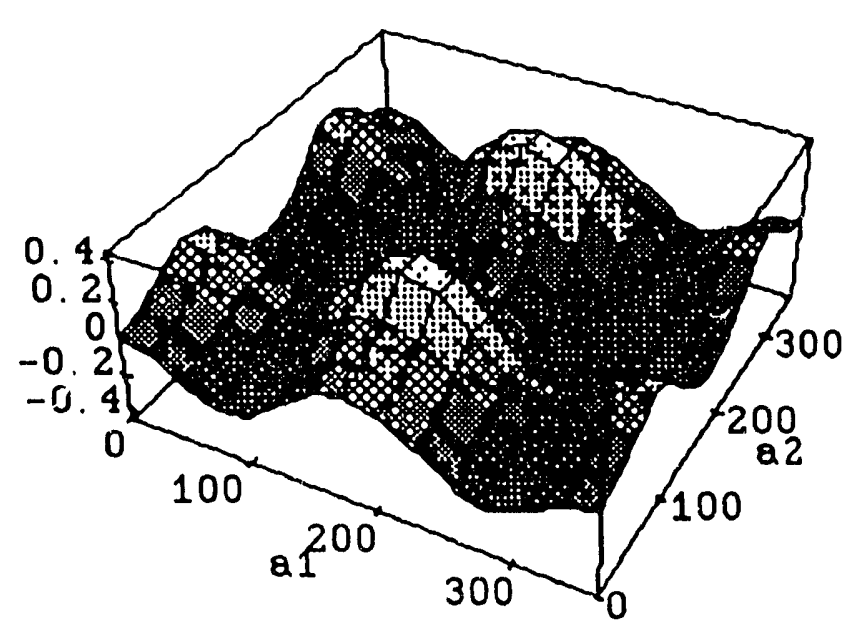

(b)

Incompatibility stresses $\sigma_{11}$ and $\sigma_{22}$ per unit of applied stress $\sigma_{33}$ for [110] asymmetrical tilt boundaries. (a) $\sigma_{11}$ (b) $\sigma_{22}$

$\operatorname{Ti}(i)$ 


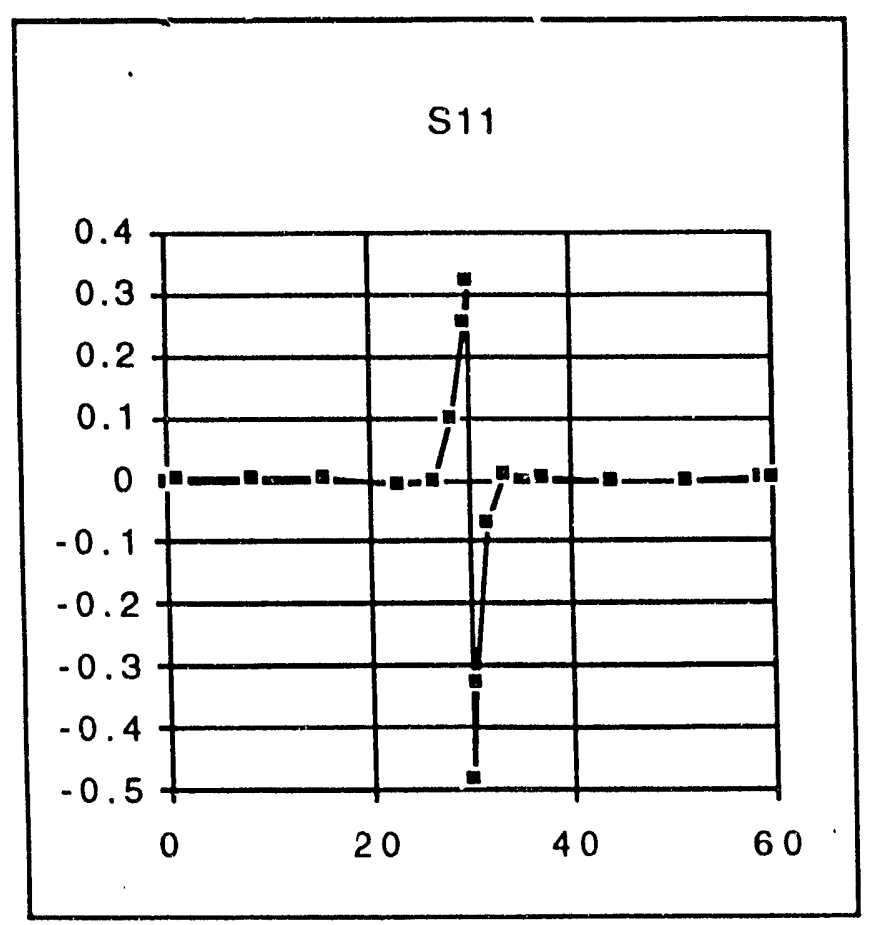

$\sigma_{11}$ axial (height/width=6)

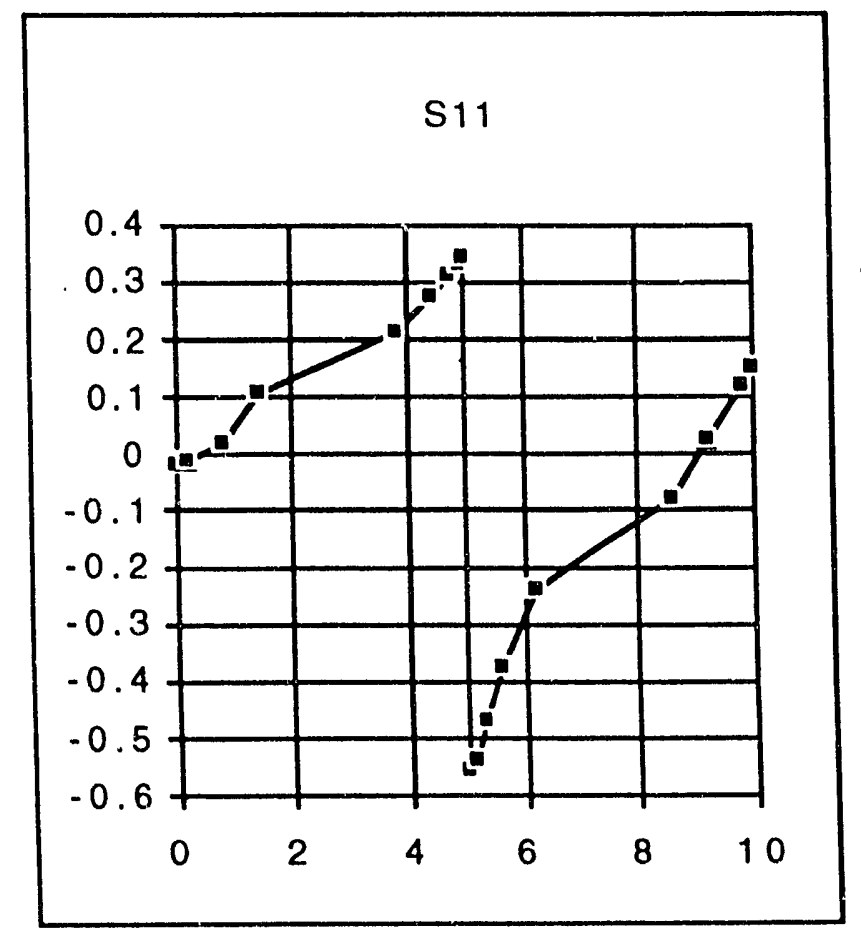

$\sigma_{11}$ axial (height/width $=1$ )

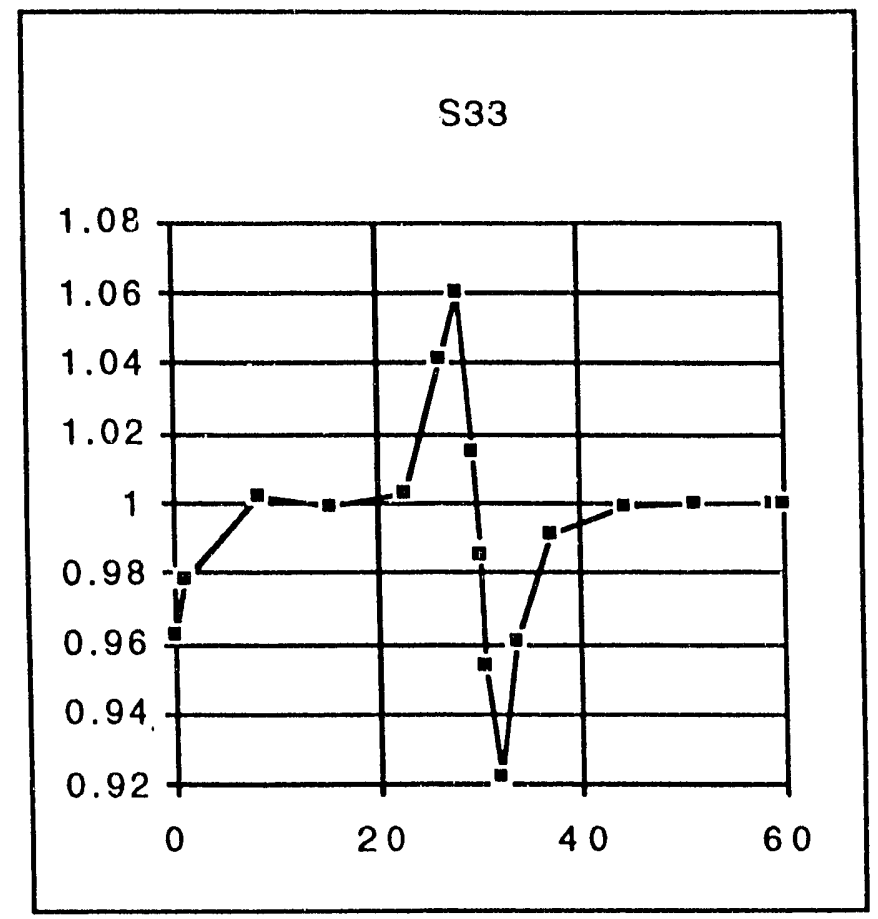

$\sigma_{33}$ axial (height/width $=6$ )

S33

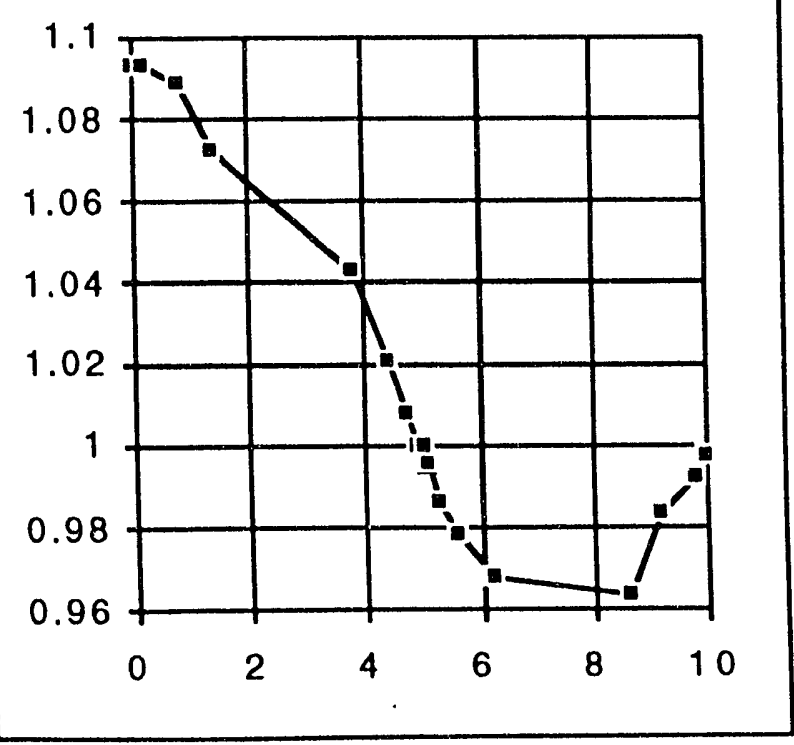

$\sigma_{33}$ axial (height/width $=1$ ) 


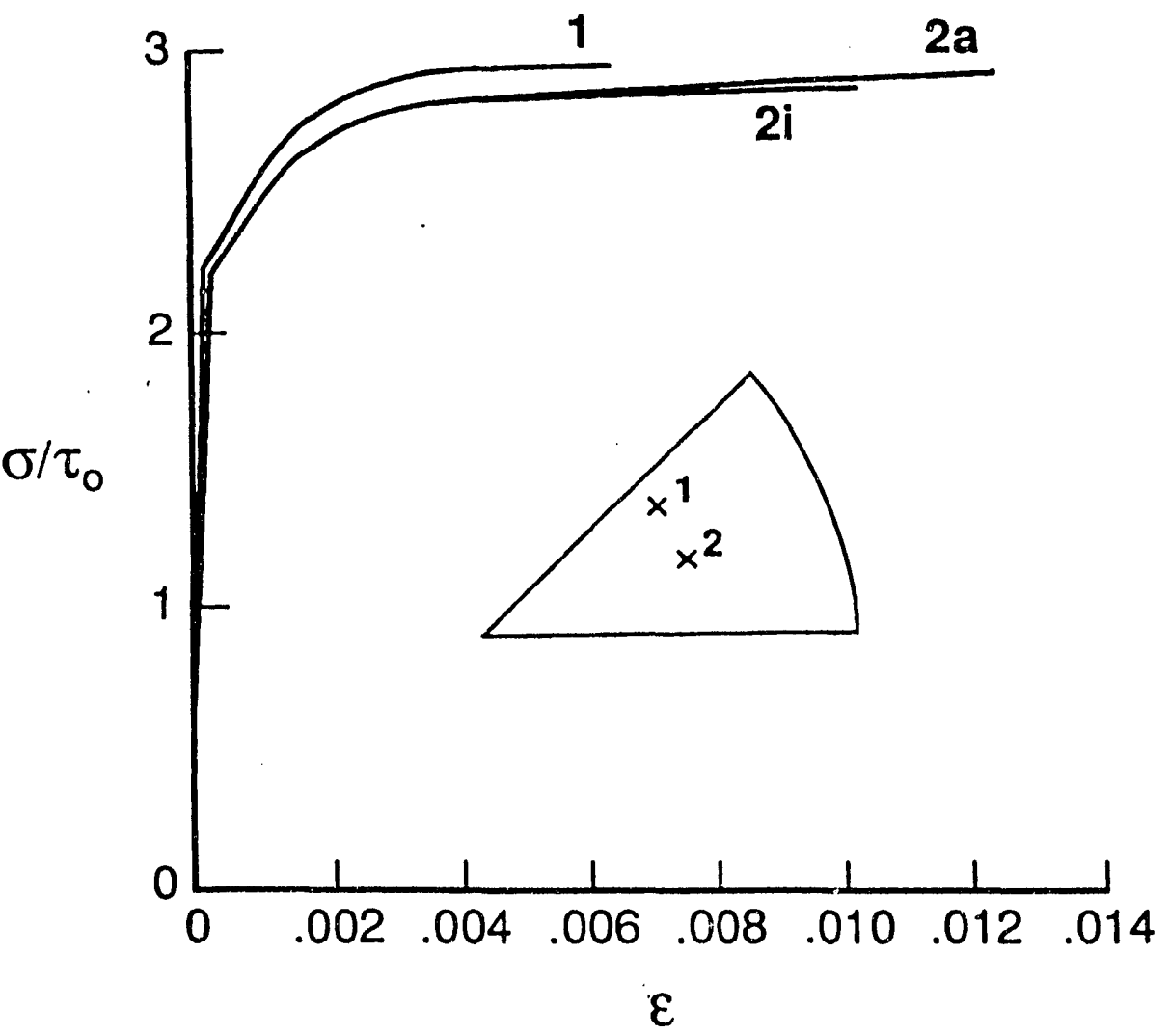

$F_{1 G} 8$ True stress-logarithmic strain curves up to coarse slip band initiation (terminus) in "single-slip" orientations. Table 3 defines parameters for each curve. 


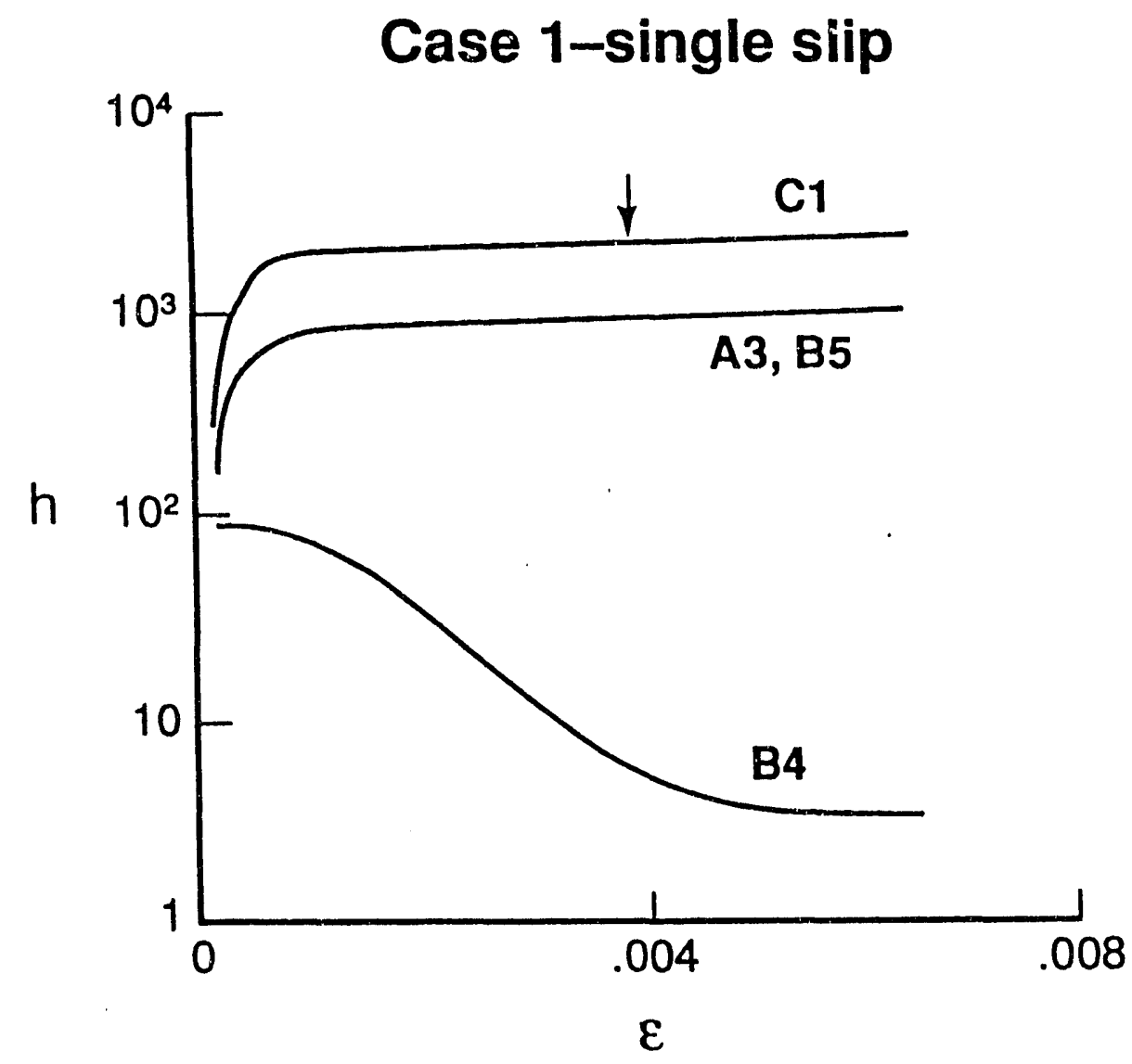

Figure 9 Evolution of the slip-system hardening moduli on the primary (B4) and secondary systems (C1), (A3), and/or (B5) corresponding, respectively, to the three cases in Fig. 18 and Table 3. a) Case 1. 


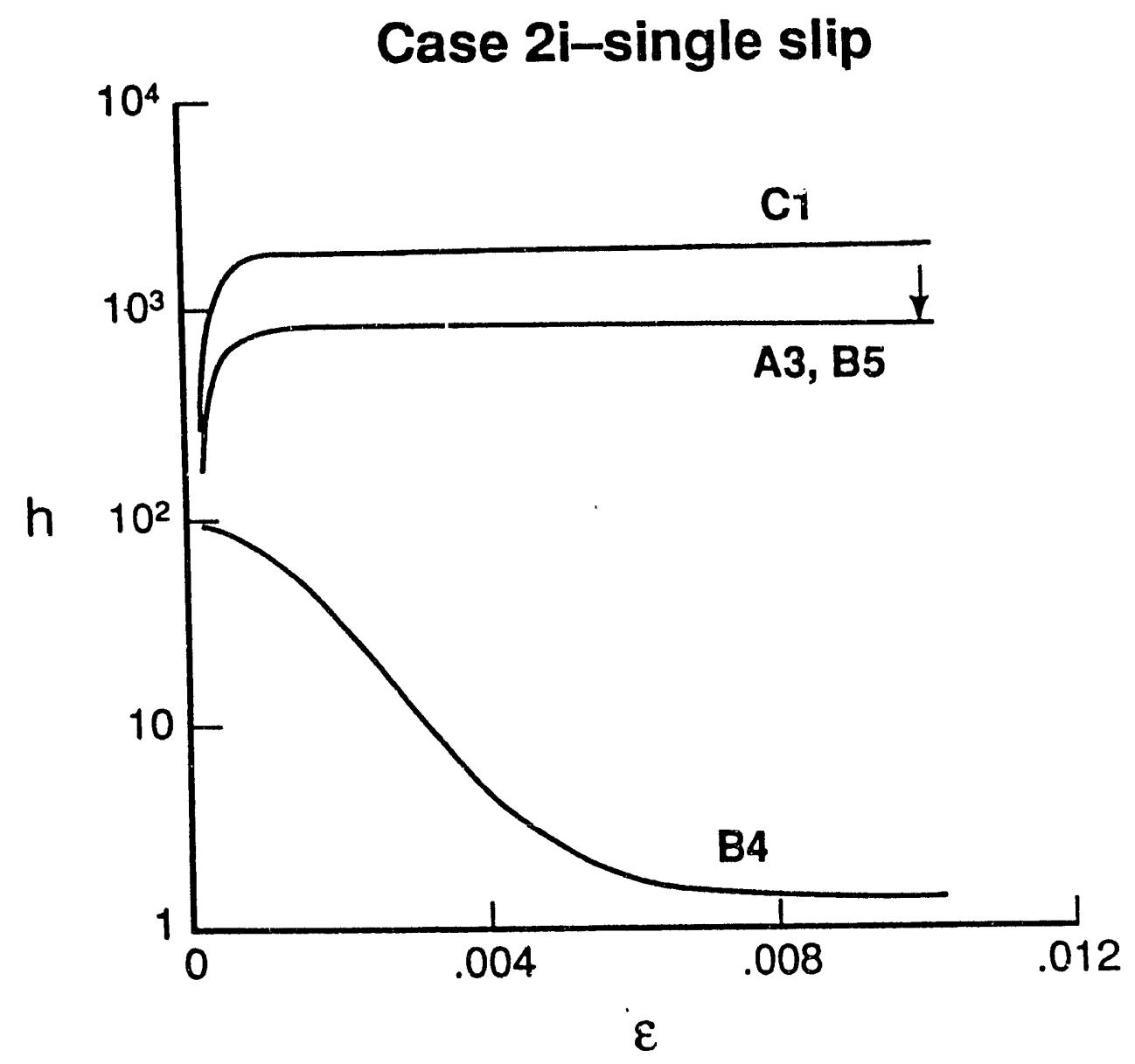

Figure $4 B$ Same as Figure 19 but for Case 2. 
Case 2a-single slip

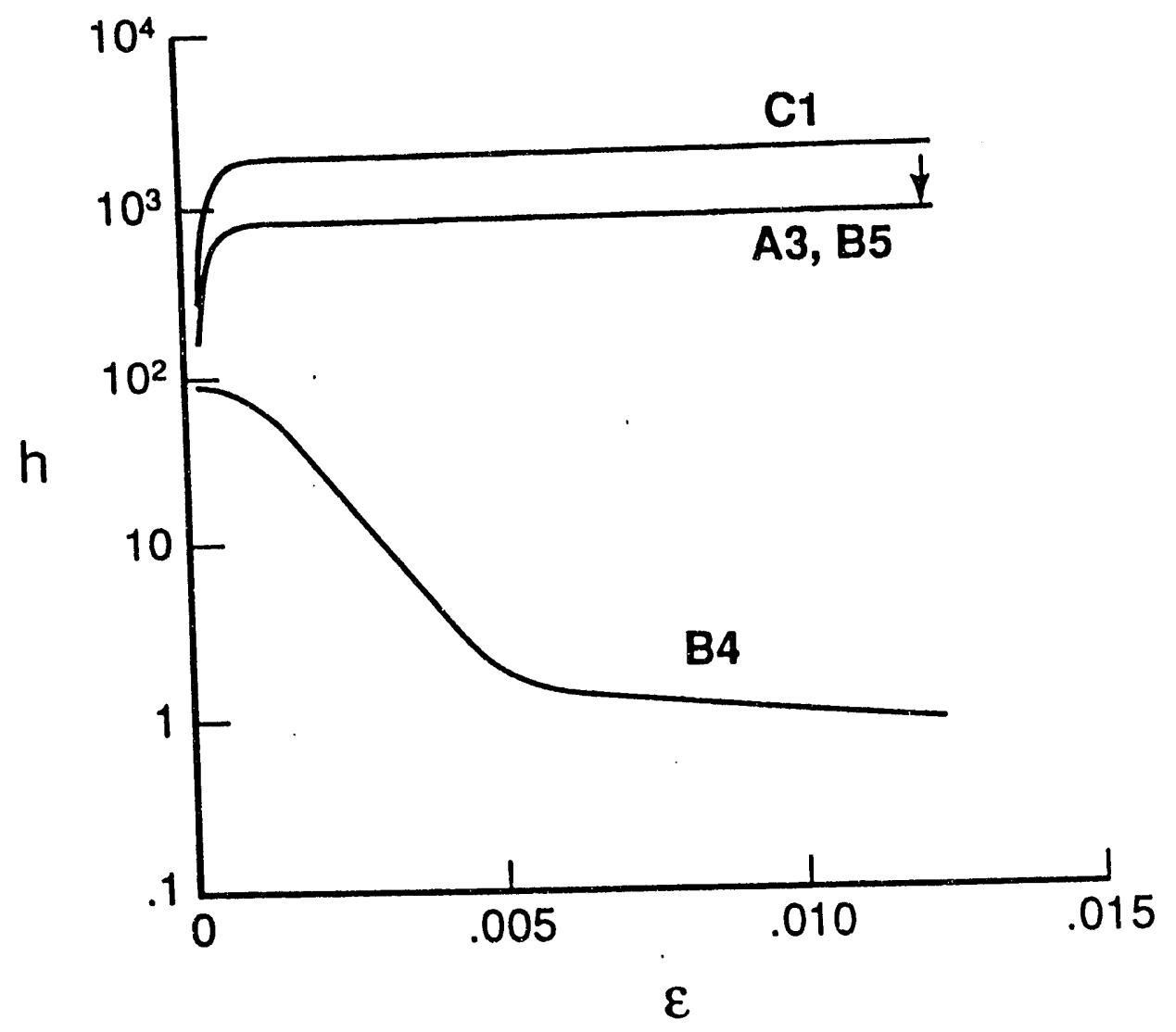

Figure $9 C$ Same as Figure 19 but for Case 3. 

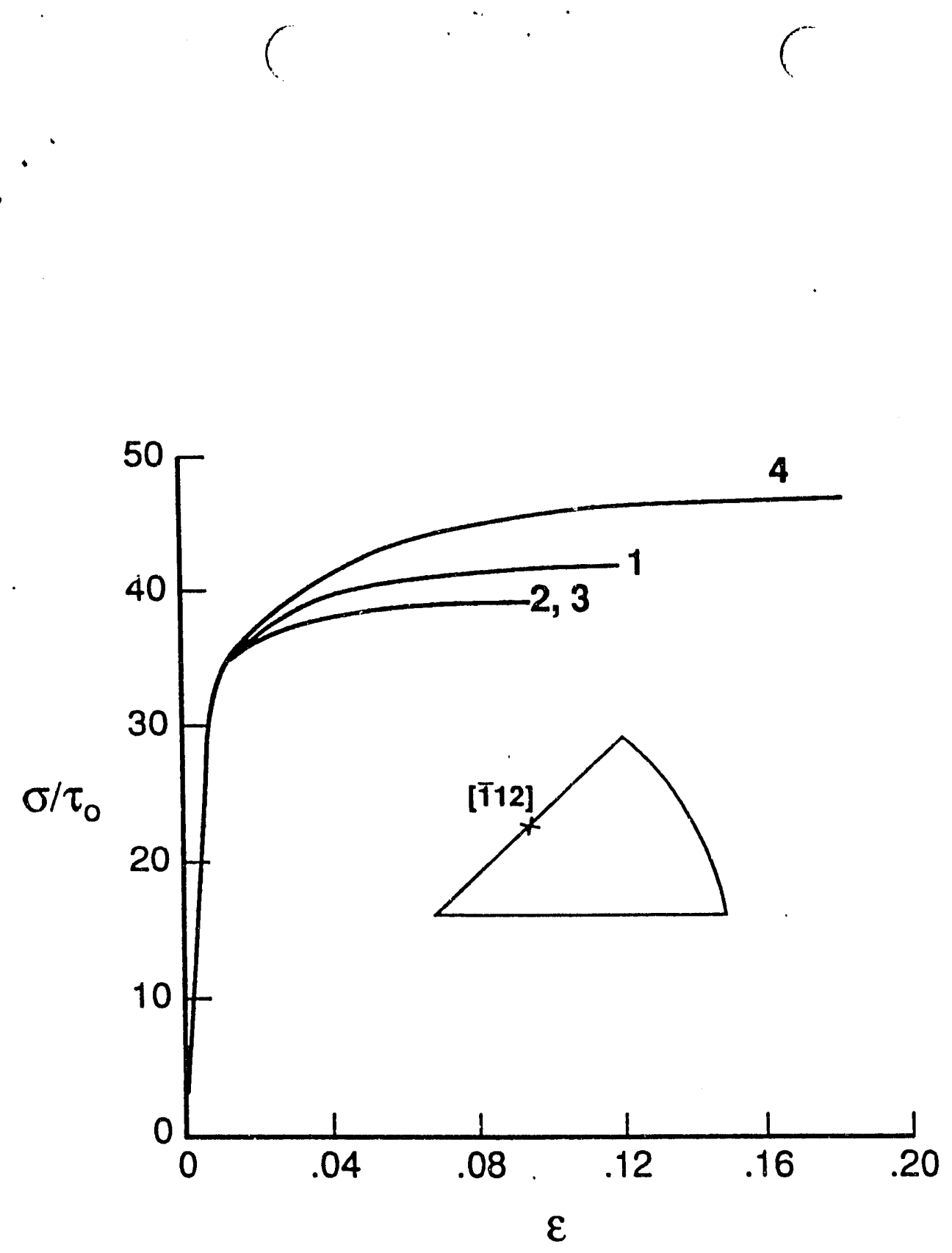

Figure 10 True stress-logarithmic strain curves up to macroscopic shear band initiation (terminus) in "double-slip" orientations. Table 4 defires parameters for each curve. 


\section{Case 1}

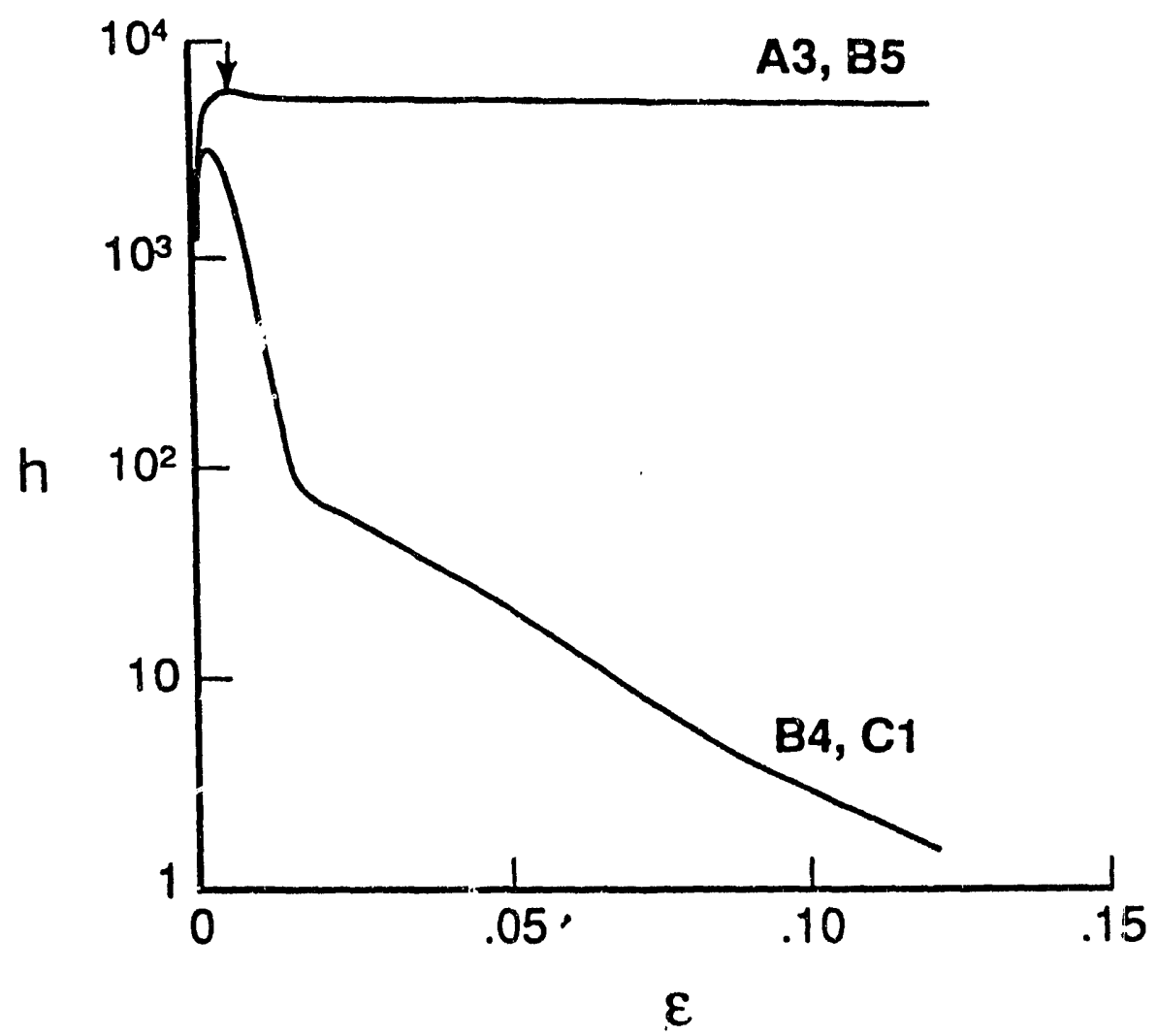

Figure // Evolution of the slip-system hardening moduli on the primary systems (B4) and (C1) and two of the secondary systems (A3) and (B5) corresponding, respectively, to the four cases in Fig. 20 and Table 4. a) Case 1. 


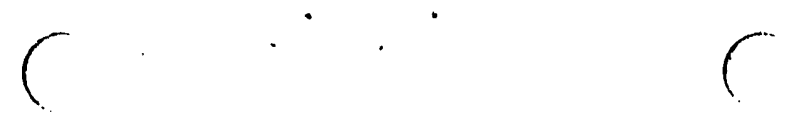

Case 2

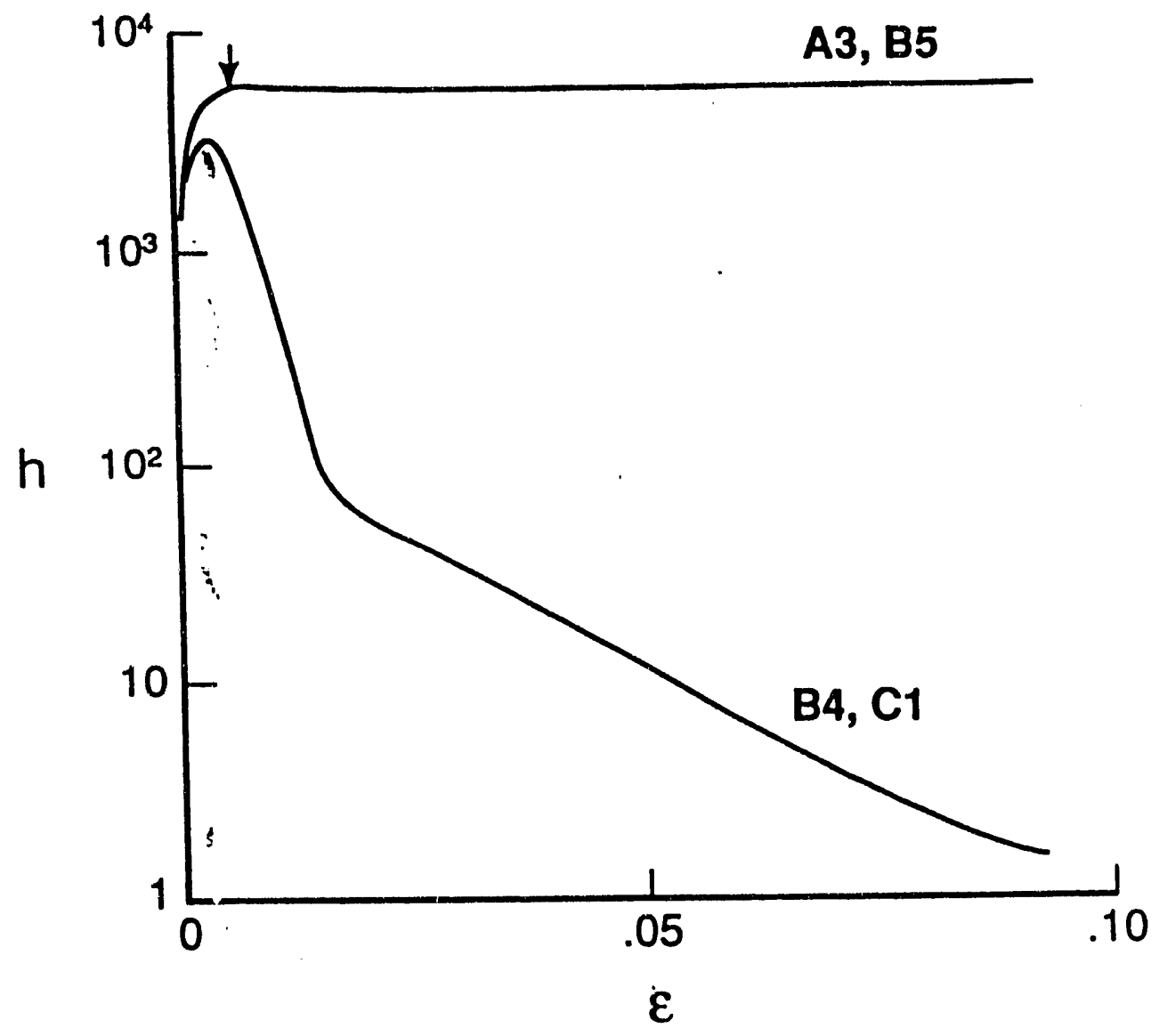

Figure $/ / B$ Same as Figure 21 but for Case 2. 
Case 3

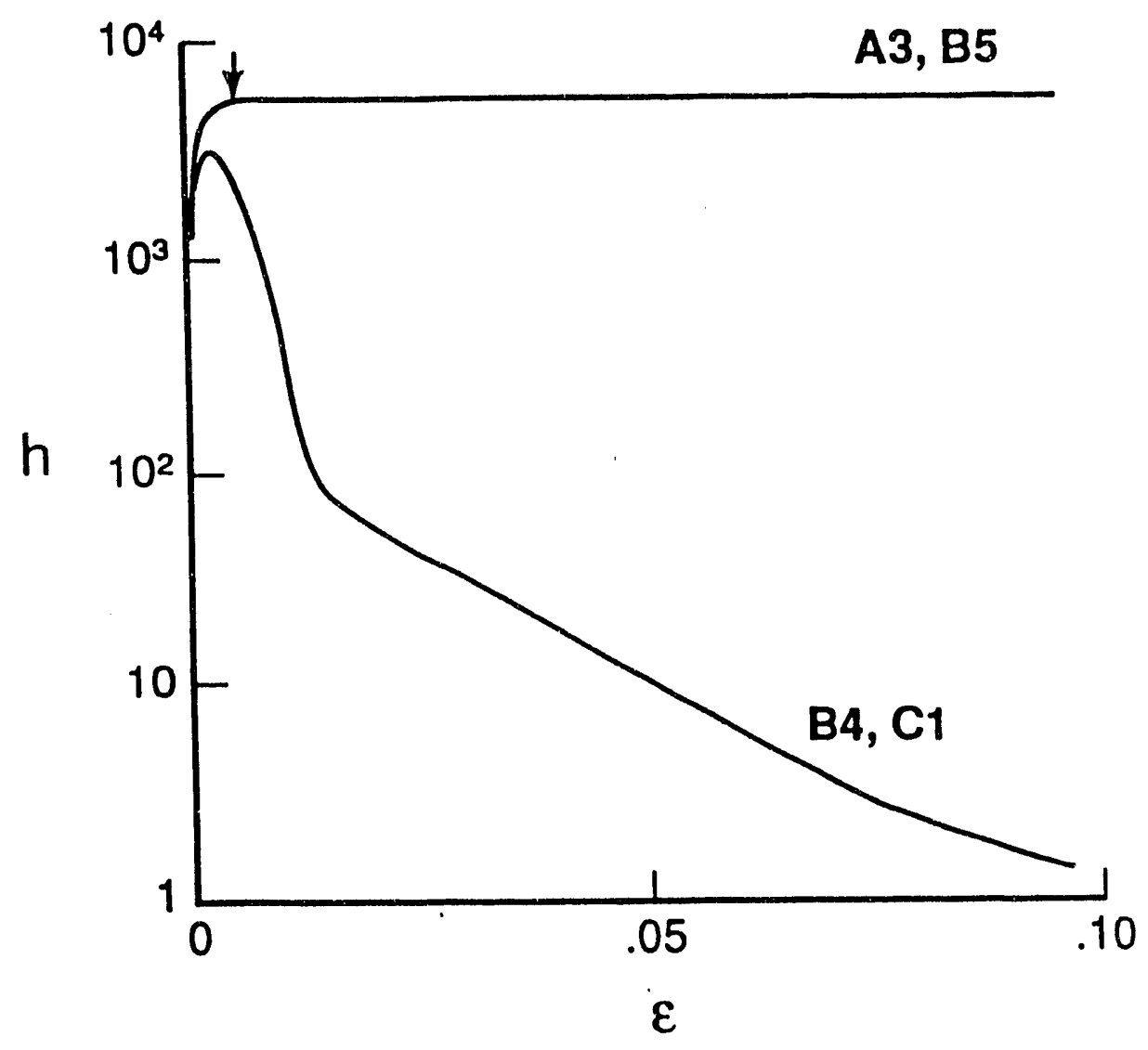

Figure $/ / C$ Same as Figure 21 but for Case 3. 
Case 4

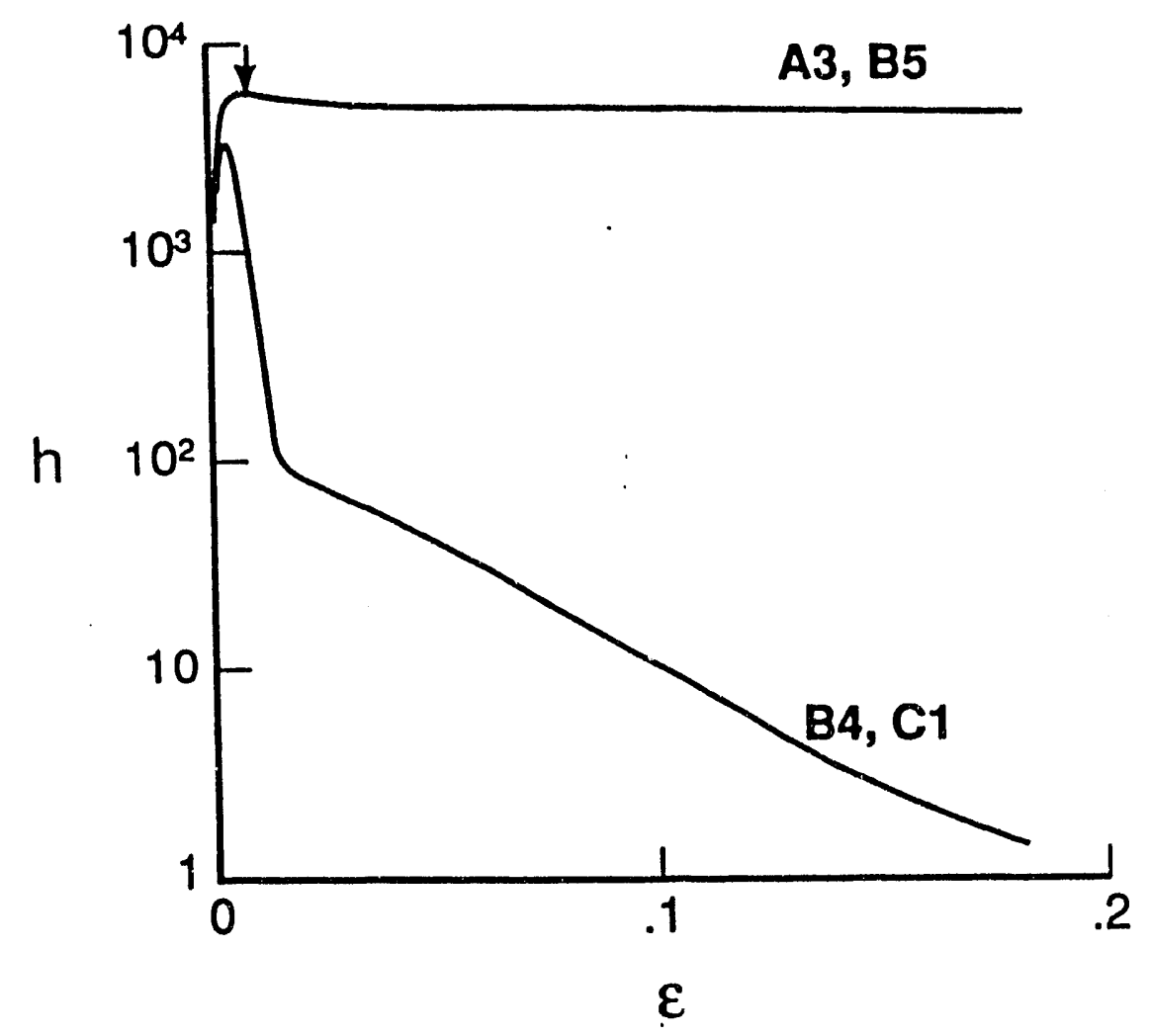

Figure // $D$ Same as Figure 21 but for Case 4. 

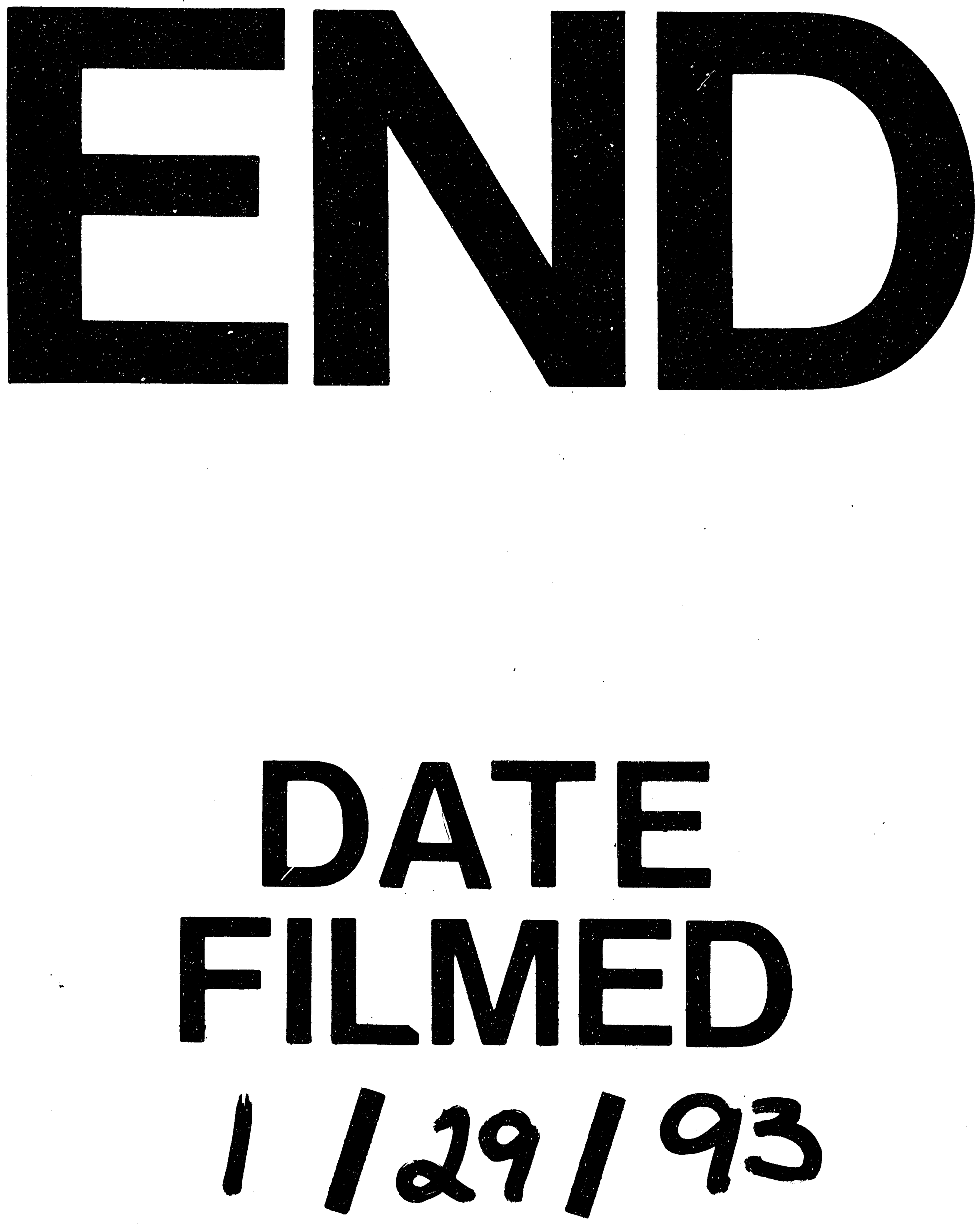
